\title{
"Esto es lo que me gusta y lo que voy a estudiar": Un estudio cualitativo sobre la toma de decisiones académicas en bachillerato ${ }^{1}$
}

\author{
"This is what I like and what I am going to study": \\ A qualitative study of academic decisions in high \\ school \\ $\mathrm{M}^{\mathrm{a}}$ del Carmen RODRÍGUEZ MENÉNDEZ, Jose Vicente PEÑA CALVO \\ y $\mathrm{M}^{\mathrm{a}}$ de las Mercedes INDA CARO \\ Universidad de Oviedo
}

Recibido: Marzo 2015

Evaluado: Mayo 2015

Aceptado: Junio 2015

\section{Resumen}

Durante la educación secundaria los estudiantes comienzan a explorar las ocupaciones y expectativas profesionales, forman sus creencias de autoeficacia y consolidan sus intereses académicos. En este contexto, el artículo presenta los resultados de una investigación realizada en el Principado de Asturias para conocer las percepciones y opiniones que tienen los estudiantes de bachillerato y sus progenitores sobre las elecciones académicas realizadas en la educación secundaria, haciendo especial hincapié en los factores contextuales que pueden ayudar u obstaculizar el desarrollo de la carrera. Se ha usado una metodología cualitativa fundamentada en el uso de la técnica del grupo de discusión. Se han organizado cinco grupos de discusión con estudiantes y dos grupos con progenitores. El total de personas que participó en las entrevistas focales fue de 51 estudiantes y de 14 padres/madres. Los resultados obtenidos nos permiten concluir que el bachillerato sigue siendo considerado la puerta de acceso a la universidad y no a la formación profesional de grado superior. También se concluye que los estudiantes fundamentan sus decisiones académicas sobre la base de la preferencia y gusto personal por determinada opciones y esta razón prevalece sobre otras como las salidas profesionales o el rendimiento académico previo. Respecto a la influencia de distintos agentes sociales en el proceso de decisión académica, los resultados son concluyentes al afirmar que el principal apoyo de los estudiantes son sus progenitores, por encima del que perciben encontrar de docentes y grupo de iguales. El artículo finaliza con algunas sugerencias para mejorar la práctica de la orientación educativa en las instituciones de educación secundaria.

Palabras clave: educación secundaria, orientación educativa, elección de estudios, análisis de contenido.

\footnotetext{
${ }^{1}$ Investigación financiada por el MICINN (EDU-2010-17233) y FONDOS FEDER.
} 


\begin{abstract}
During high school students begin to explore occupations and professional expectations, form their self-efficacy beliefs and strengthen their academic interests. In this context, the paper presents the results of a research conducted in the Principality of Asturias to know high school students' and their parents' perceptions and opinions about academic choices made in secondary education, with particular emphasis on contextual factors that may help or hinder vocational development. We have used a qualitative methodology based on the use of the focus group. We have organized five focus groups with students and two focus groups with parents. The total number of people who participated in the focus group was 51 students and 14 fathers / mothers. The results obtained allow us to conclude that high school is still considered the gateway to university and not to vocational education and training studies (VET). It is also concluded that students base their academic decisions on personal taste and preference for certain options and this reason prevails over others such as professional opportunities or prior academic achievement. Regarding the influence of different social agents in the process of academic decision, the results are conclusive to say that their parents are the main support of the students. The article concludes with some suggestions for the improvement of educational guidance in institutions of secondary education.
\end{abstract}

Keywords: secondary education, educational guidance, choice of studies, content analysis.

La toma de decisiones académicas en la educación secundaria ha sido objeto de estudio de la literatura científica en el ámbito de la orientación para la carrera. En este sentido, cuando un estudiante toma una decisión importante que condicionará su futuro académico y profesional debería de hacerlo evitando la improvisación y sopesando los pros y contras de cada una de las posibilidades que tiene a su alcance (Santana, Feliciano y Jiménez, 2009). Además, en este proceso el estudiante debe contar con el apoyo de los agentes de socialización (progenitores, docentes) que tienen más influencia en su desarrollo personal.

En este contexto, la investigación que presentamos ha sido realizada en el Principado de Asturias para conocer las percepciones y opiniones que tienen los estudiantes de bachillerato, progenitores y docentes sobre las elecciones académicas que se realizan en la educación secundaria, haciendo especial hincapié en el papel de los distintos agentes de socialización en esta elección. En el artículo presentamos, fundamentalmente, las valoraciones de los estudiantes; no obstante, también daremos voz a padres/madres para corroborar algunos de los resultados más destacados del estudio.

\title{
Marco teórico
}

El proceso de decisión vocacional es complejo y en él influyen multitud de factores. Desde este punto de vista, un aspecto fundamental a destacar son las motivaciones personales que provocan que un adolescente elija una determinada opción académica. Sobre esta cuestión, Santana y Feliciano (2009) nos indican que los estudiantes de su muestra de bachillerato fundamentan sus decisiones vocacionales en criterios personales tales como sus gustos, la propia capacidad o la necesidad de hacer algo de 
lo que sentirse orgullosos. En línea similar, Cortés y Conchado (2012) concluyen que los estudiantes de bachillerato afirman que es la vocación el principal factor que condiciona su toma de decisiones por encima de agentes sociales, aunque estos agentes también juegan un papel relevante. Del mismo modo, García, Padilla y Suárez (2009) concluyen que las chicas de su investigación justifican, en la mayoría de los casos, sus elecciones académicas en virtud de algún contenido del campo profesional que les resulta atractivo; añadiendo que las alumnas son poco elocuentes al argumentar y justificar sus elecciones.

En suma, los estudios parecen indicar una toma de decisión fundamentada en criterios personales como el gusto o la vocación; no obstante, también explican que distintos agentes sociales pueden mediar en la decisión. Por ello, en la etapa de la adolescencia también es importante tener en cuenta los apoyos y las barreras del contexto social que rodea al estudiante para entender sus decisiones académicas. Los niveles de apoyo de los padres/madres, demás familiares, compañeros/compañeras y docentes pueden influir en sus planes educativos y en sus aspiraciones profesionales.

La mayoría de los estudios realizados, tanto en España como en otros países, han analizado el papel que juegan los progenitores en el proceso de elección académica. En este sentido, el estudio realizado por Santana y Feliciano (2009, 2011. Véase también Santana, Feliciano y Jiménez, 2009) constató que la alternativa académico-laboral por la que opta un estudiante de Bachillerato está influenciada por las valoraciones y apoyos percibidos en su medio familiar. De forma similar, en el estudio de Cortés y Conchado (2012) el 74.6\% de los estudiantes seleccionaron a sus padres/madres como las personas más influyentes respecto a la cuestión de qué harían al finalizar los estudios de bachillerato. De este estudio también se destaca que los progenitores no suelen interponerse en las decisiones de sus hijos e hijas y que, incluso cuando la opción elegida no era la que más les satisfacía, la mayoría aceptaban la decisión de los estudiantes.

Diversos estudios realizados en el ámbito anglosajón confirman estos resultados. Así, Keller y Whiston (2008) indican que el apoyo parental es un factor clave en el desarrollo de la carrera de los estudiantes de secundaria. Se concluyó que los adolescentes necesitan saber que sus padres/madres se interesan por ellos, creen en sus capacidades y confían en que sabrán tomar buenas decisiones (véase también Schultheiss et al., 2001). En línea similar Fisher y Padmawidjaja (1999) afirman que los estudiantes de secundaria reconocen el valor de que sus padres/madres estén siempre accesibles a sus requerimientos y reconocen la importancia de su asesoramiento y de que no les fuercen a elegir determinadas opciones académicas. Asimismo, en el estudio de Perrone et al. (2002) se concluyó que los modelos de rol más comúnmente citados por los estudiantes en su proceso de decisión fueron los padres/madres, especialmente las madres para las chicas y los padres para los chicos.

El estudio cualitativo de Schultheiss et al. (2001) reconoce la importancia de que los progenitores estimulen a sus hijos e hijas a explorar las distintas opciones que tienen, a aprender de los errores cometidos y a tomar sus propias decisiones académicas. De forma complementaria, en el estudio cualitativo de Hill, Ramírez y Dumka (2003) los estudiantes de familias que proveen escaso o nulo apoyo tienden a 
no tener metas claramente definidas y a percibir mayores barreras en sus aspiraciones académicas. Finalmente, destacamos el estudio de Otto (2000) que concluye que la mayoría de los estudiantes de su muestra declaran que dialogan con sus padres/madres respecto a sus planes vocacionales algunas veces o frecuentemente. Asimismo, se indica que eran sus madres las que eran más conscientes de sus intereses y habilidades, y las que más les ayudaban cuando hablaban sobre sus planes de carrera (véase también Nauta y Kokaly, 2001; Wycoff, 1996).

De forma complementaria, otros estudios también reconocen el importante papel desempeñado por el grupo de iguales en el proceso de decisión vocacional. En este sentido, una posible explicación a esta influencia es que los amigos son modelos de rol muy accesibles porque se comparten similares intereses y valores. Además, pueden ser mejores fuentes de información académica que los progenitores y juegan un papel importante proporcionando apoyo emocional y reforzando sentimientos de confianza y seguridad (Ali, McWhirter y Chronister, 2005; Dietrich y Kracke, 2009; Kenny et al., 2003; Ma y Yeh, 2010; McWhirter, Torres, Salgado y Valdez, 2007; Schultheiss et al., 2001). Igualmente, en la investigación de Otto (2000) el $80 \%$ de los adolescentes indican que hablan seriamente con sus amigos sobre sus planes académicos y el $57 \%$ lo hacen respecto a la formación académica necesaria para acceder a la ocupación deseada.

Respecto al papel de los docentes, investigaciones recientes en nuestro país demuestran que los estudiantes de bachillerato no citan a los docentes entre las personas que les ayudan a tomar decisiones al final del bachillerato, demostrándose la poca incidencia de la acción tutorial para ayudar a identificar la relación entre las características personales y las de las profesiones y para aclarar dudas sobre el futuro académico (Santana y Feliciano, 2008). En este sentido, estos resultados no son coincidentes con los obtenidos en el contexto anglosajón, pues diversas investigaciones han concluido que los estudiantes perciben a los docentes como sus mentores principales, una vez excluidos los miembros adultos de su grupo familiar (Flores y Obasi, 2005). De forma complementaria, en el estudio de Nauta y Kokaly (2001) el $31 \%$ de los estudiantes de su muestra declararon que una profesora fue su mayor influencia escolar en el proceso de toma de decisión vocacional, mientras que para el $25 \%$ lo fue un docente varón. Asimismo, en un estudio cualitativo realizado con estudiantes, familias y docentes de educación secundaria se reconoció el papel de los docentes, a través de su apoyo y asesoramiento, en la orientación académicoprofesional (Carvalho y Taveira, 2014). También destacamos el estudio de Fouad et al. (2010) donde se concluyó que la percepción que tenían los estudiantes de lo que los profesores de matemáticas y ciencias esperaban de ellos fue un apoyo consistente para su continuidad en estas materias.

Finalmente señalamos el estudio de Paa y McWhirter (2000) que analiza la influencia de distintos agentes socializadores en la toma de decisión vocacional. Sus resultados más destacados fueron que las personas con una mayor influencia sobre las estudiantes eran sus madres, padres y amigas, por este orden. Profesores y orientadores eran quienes tenían un peso menor en la decisión académica. Para los chicos la mayor 
influencia era ejercida por el padre, la madre y los amigos. Mientras profesoras y amigas eran las que tenían un menor peso en la decisión.

A partir de estos planteamientos teóricos, indicamos que nuestra investigación ha tenido como objetivo fundamental analizar los factores que influyen en el proceso de decisión vocacional de los estudiantes de bachillerato, haciendo especial hincapié en las barreras y apoyos sociales que tienen en el proceso.

\section{Método}

\section{Participantes}

Los resultados que presentamos se corresponden con la segunda fase de un estudio emprendido por el grupo de investigación ASOCED. En la primera fase de la investigación se administró un cuestionario a 2359 estudiantes de $4^{\circ}$ de la ESO y a 1558 estudiantes que cursaban el primer curso del bachillerato científico-tecnológico. En esta primera fase se obtuvo información precisa sobre el proceso de decisión académica, especialmente sobre los factores que influían en este proceso. Una vez identificados dichos factores, el equipo de investigación abordó la realización de una segunda fase de la investigación para conocer la percepción que tenían los distintos implicados sobre el proceso de decisión académica. En este sentido, se decidió que era imprescindible dar voz a los distintos participantes para que contaran, de primera mano, sus vivencias, sensaciones, opiniones y sentimientos. En esta segunda fase han participado 51 estudiantes ( 23 chicas y 28 chicos), 14 padres/madres (12 madres y 2 padres) y 38 docentes (23 mujeres y 15 varones). Los estudiantes estaban cursando el bachillerato de la modalidad científico-tecnológica y los docentes impartían docencia en esta modalidad académica.

\section{Instrumento de recogida de datos}

La recogida de datos se realizó mediante la técnica del grupo de discusión pues este instrumento crea un ambiente en el que los distintos participantes son estimulados a intervenir activamente en el diálogo por las ideas y opiniones que presentan los demás, incrementando la calidad de los datos recogidos. Asimismo, es una técnica que permite la exploración de las experiencias de los participantes porque pueden expresar sus propias opiniones y abordar los temas desde su punto de vista.

Se organizaron cinco grupos de discusión con estudiantes que cubrieron el espectro geográfico del Principado de Asturias. Uno de los grupos de discusión se realizó en el occidente asturiano, otro en el oriente asturiano, dos en la zona central y uno en la cuenca minera. Además, cuatro de los grupos de discusión se realizaron en Institutos de Educación Secundaria y uno de ellos en un colegio concertado. Asimismo, se realizaron seis grupos de discusión con docentes en los mismos centros en los que se realizaron los grupos focales con estudiantes, añadiéndose un grupo más en la zona central de Asturias. Por último, se realizaron dos grupos con padres/madres, uno en 
Oviedo y otro en la cuenca minera. La media de asistentes a los grupos de discusión se situó entre las seis y las ocho personas.

El protocolo de preguntas que se usó ha sido desarrollado por el grupo de investigación después de realizar una amplia revisión bibliográfica y de analizar las principales conclusiones obtenidas en la primera fase del estudio. Se usaron diferentes protocolos con los distintos colectivos, si bien las temáticas que se plantearon para la discusión fueron similares. En el caso de los estudiantes se debatieron los siguientes temas: elección de asignaturas y rendimiento académico, asignaturas y creencias de autoeficacia, interés por distintas asignaturas, percepción de los apoyos y las barreras que encuentran en su elección académica (de docentes, progenitores, iguales, otras), elección de estudios y estereotipos de género. Los grupos de discusión con los progenitores ahondaron en los siguientes aspectos: elección académica de sus hijos e hijas; percepción del éxito académico de sus hijos e hijas; utilidad de las distintas asignaturas para su futuro profesional; apoyo mostrado a sus hijos e hijas en el proceso de decisión académica; asignaturas y estereotipos de género.

\section{Procedimiento}

A partir de los contactos previos con setenta y dos centros de educación secundaria del Principado de Asturias para realizar la primera fase de la investigación, acudimos a aquellos que tuvieron interés por participar en la segunda fase. Además, se cuidó de que hubiera una representación de centros de distintas titularidades (pública y concertada) y también se respetó que los centros estuviesen en diferentes zonas geográficas del Principado de Asturias. Una vez que los centros accedían a participar, los grupos de discusión se llevaron a cabo en los centros de enseñanza y en el horario establecido por el centro para tal fin. Aunque los participantes conocían el objetivo para el que habían sido convocados, las reuniones comenzaron con una exposición de nuestros objetivos. Presentábamos nuestra investigación como un estudio diagnóstico sobre los procesos de toma de decisión que seguían los estudiantes de secundaria cuando debían afrontar elecciones académicas. Los grupos de discusión fueron conducidos por dos miembros del equipo de investigación.

Creemos haber logrado una relación de confianza y respeto mutuo con los participantes. En líneas generales, la discusión surgió de forma fluida porque el tema se prestaba al debate. Todas las sesiones fueron grabadas contando con la aprobación de los participantes. Con posterioridad, estas cintas fueron transcritas en papel. De forma complementaria a la grabación, uno de los entrevistadores participantes en la sesión tomó notas durante la entrevista grupal para proveer información de contexto que ayudara a interpretar el contenido de las trascripciones durante el análisis de contenido. 
$\mathrm{M}^{\mathrm{a}}$ del Carmen Rodríguez Menéndez et al. Esto es lo que me gusta y lo que voy a estudiar”...

\section{Análisis de datos}

El trabajo con grupos de discusión requiere el uso de una aproximación analítica, propia de una metodología cualitativa, que se basa en el análisis de contenido (Valles, 2000). Con este propósito, se establecieron tres niveles de análisis de los datos:

1. Clasificación temática de las distintas ideas verbalizadas en los grupos de discusión, elaborando, a partir de ello, un sistema de categorías. Cada entrevista grupal fue inicialmente codificada en conceptos que luego fueron comparados entre sí con objeto de generar grupos más amplios de categorías. Se identificó, localizó y comparó toda la información obtenida, extrayendo discursos recurrentes y buscando una denominación común a los distintos fragmentos de discurso.

2. Descripción del contenido de las trascripciones: inclusión de comentarios de los participantes en relación con la categoría trabajada en cada momento. Estos comentarios fueron elegidos por su claridad expositiva, su significatividad en relación al tópico expresado y porque presentaban matices específicos que enriquecían el análisis y evitaban la redundancia.

3. Interpretación teórica del contenido descrito en el segundo nivel de análisis. Se elaboraron conclusiones en relación a los objetivos de la investigación y tomando como punto de referencia los desarrollos teóricos efectuados, así como los resultados de investigaciones similares.

En la figura 1 se plasma el sistema de categorías elaborado respecto a los datos presentados en este artículo.

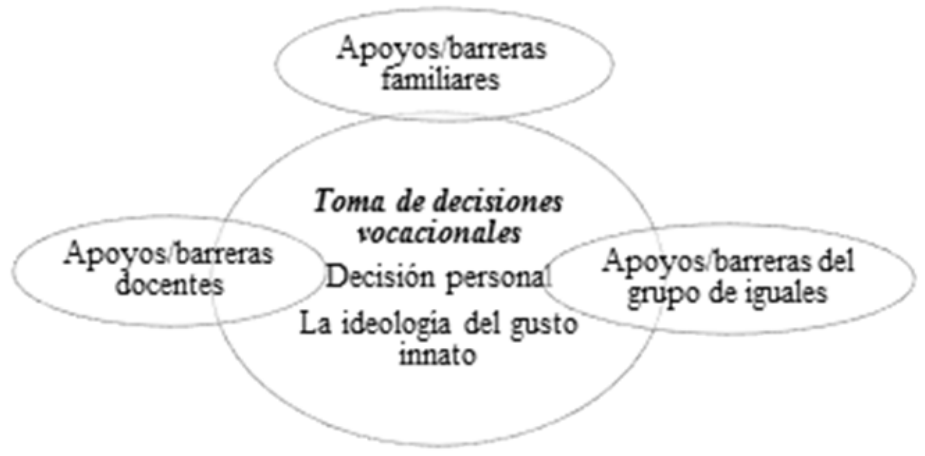

Figura 1. Sistema de categorías elaborado a partir del análisis de contenido

\section{Resultados}

\section{“Elijo lo que me gusta”: la elección es una decisión personal}

Todos los estudiantes de nuestra muestra siguen considerando que el bachillerato es su puerta de acceso a los estudios universitarios. Solamente un estudiante se mostró indeciso ante la posibilidad de realizar un módulo de grado superior, si bien 
$\mathrm{M}^{\mathrm{a}}$ del Carmen Rodríguez Menéndez et al. Esto es lo que me gusta y lo que voy a estudiar”...

finalmente, y condicionado por la opinión de sus docentes, se ha decantado por proseguir sus estudios en la universidad. Por otro lado, la razón fundamental que indican los estudiantes que cursan Bachillerato científico-tecnológico para elegir esta modalidad tiene que ver con lo que hemos denominado como la "ideología del gusto innato". Según su opinión han elegido estos estudios porque les gustan y afirman que el gusto es algo "que se tiene", no algo construido socialmente a partir de las diversas influencias sociales a las que pueden estar sujetos. Esta idea conecta con otra de la que se hablará más adelante y que tiene que ver con la convicción de que la elección siempre es individual y personal, pese a los apoyos sociales que puedan tener. Así, es muy frecuente que usen expresiones que enfatizan una relación de afinidad con determinadas materias: "siempre me gustó", "nunca me gustó". También observamos que algunos estudiantes usan la expresión "no me veo" o "no me pega" para denotar su escasa o nula preferencia por determinados campos académicos y profesionales. Estas ideas también son confirmadas por los progenitores que usan el mismo tipo de expresiones para referirse a las preferencias académicas de sus hijos e hijas:

El tecnológico porque engloba las actividades profesionales que me gusta desarrollar: mecánica, automoción, electricidad, cosas manuales (Varón, GD1) ${ }^{2}$.

Escogí el tecnológico porque me gustan más las ingenierías que biología o medicina, ese tipo de carreras no me atraen (Varón, GD5).

No me gusta lo manual y las ingenierías son todo de manual (Mujer, GD1).

No me veo de enfermero (Varón, GD1).

Mi hijo eligió el tecnológico porque yo creo que nació ya siendo así, porque siempre dijo de pequeño que quería trabajar con las manos. Y lo eligió él porque quiso y porque le gusta (Mujer, GD1).

Mi hijo eligió esto porque le gusta y porque es lo que se le da bien (Mujer, GD1).

En otras ocasiones, y normalmente vinculado a la ideología del gusto innato, algunos estudiantes comentan que su elección también ha estado condicionada por las salidas profesionales de la modalidad:

Tampoco sé muy bien lo que hacer pero elegí la rama sanitaria porque creo que tiene más salidas hacia más cosas (Mujer, GD3).

Escogí el tecnológico porque sentía que tenía más facilidad para este tipo de asignaturas y porque me interesan más las salidas (Varón, GD5).

En líneas generales, los estudiantes no manifiestan indecisión en sus metas académicas. Debemos tener presente que son estudiantes de Bachillerato con un perfil académico definido y que saben cuáles son las opciones que pueden elegir y, de hecho,

\footnotetext{
${ }^{2}$ Cuando se inserten citas textuales de los discursos se incluirá, entre paréntesis, una anotación que contiene el género de la persona y el grupo de discusión en el que participó.
} 
$\mathrm{M}^{\mathrm{a}}$ del Carmen Rodríguez Menéndez et al. Esto es lo que me gusta y lo que voy a estudiar”...

la elección ya está tomada, aunque al curso siguiente la puedan cambiar. Otro aspecto diferente es el relativo a las razones que fundamentan su elección, pues de los grupos de discusión se deduce que, con frecuencia, dichas elecciones no están fundamentadas en un proceso de toma de decisiones racional, sopesando los pros y contras de cada elección, simplemente eligen porque les gusta. Debemos comentar que algunos padres y madres no tienen tan claro esa seguridad en la elección y manifiestan sus dudas de que esté fundamentada en criterios racionales. Asimismo, sostienen que sus hijos e hijas no son capaces de argumentar el porqué de su elección, dando la sensación de que se trata de una idea preconcebida que no obedece a ninguna razón que sea posible explicar:

Mi hija es un mar de dudas total, no sabes ni por qué (Varón, GD1).

Entonces no veo que tengan muy claro todavia lo que quieren. Yo creo que tienen esa nebulosa de un mar de dudas increible (Mujer, GD2).

Los cambios no van de mes en mes van de cuarto de hora en cuarto de hora (Mujer, GD1).

El mío dice que quiere hacer una ingeniería y lo tiene metido en la cabeza desde que era pequeño, y yo tampoco entiendo muy bien porqué. Muchas veces lo hablamos y no es capaz de explicar por qué. Razonar no lo razona.... simplemente como una fijación que tiene (Mujer, GD1).

Un aspecto a destacar respecto a los que acabamos de comentar es la relación que establecen los estudiantes entre finalizar con éxito sus estudios universitarios y el interés por dichos estudios. Así, es muy frecuente que los estudiantes, tanto chicos como chicas, realicen la interpretación de que creen que terminarán la carrera sin mucho esfuerzo porque al estudiar lo que les gusta, el interés será mayor y el esfuerzo requerido menor. Desde nuestro punto de vista, es una concepción poco realista, máxime cuando algunas de las carreras que van a elegir (medicina, ingenierías) se caracterizan por tener que dedicar mucho tiempo y esfuerzo para aprobar:

Yo creo que la carrera, si te gusta, que te va a costar mucho menos que el bachiller (Mujer, GD1).

Creo que no me resultará dificil la carrera porque das lo que te gusta (Varón, GD2).

Es lo que me gusta y si haces algo que te gusta es más llevadero y más sencillo de hacer (Varón, GD5).

Apoyos y barreras familiares percibidos en el proceso de decisión vocacional

Lo primero que debemos destacar es que, sin lugar a dudas, los progenitores son los principales apoyos de los estudiantes: 
$\mathrm{M}^{\mathrm{a}}$ del Carmen Rodríguez Menéndez et al. Esto es lo que me gusta y lo que voy a estudiar’...

Mi madre es la que siempre me apoya en todo. El mejor apoyo que tengo (Mujer, GD1).

Tus padres siempre te van a apoyar y quieren lo mejor para ti (Varón, GD3).

Confían y me apoyan para que saque el bachillerato (Varón, GD4).

De forma generalizada se expresa la opinión de que padres y madres son un apoyo fundamental en el desarrollo académico de chicos y chicas. No obstante, también es muy frecuente que el alumnado considere que su proceso de elección vocacional ha sido algo muy personal, es decir, los padres/madres apoyan las decisiones que ellos han tomado pero la decisión última es realizada "en solitario" por los estudiantes, o al menos es así como ellos y ellas lo perciben. Chicos y chicas consideran que su elección está basada en su gusto personal por determinadas materias y opciones académicas; en consecuencia, la decisión de cursarlas es individual, la persona se enfrenta "sola" a su toma de decisiones. A lo más, los progenitores ponen una condición, que estudien el bachillerato $\mathrm{y}$, a ser posible, una carrera universitaria. Los progenitores tienen expectativas académicas sobre sus hijos e hijas y, en cierto modo, han apoyado la continuidad y perseverancia en sus estudios. Los estudiantes perciben que los progenitores son agentes secundarios que apoyan una decisión previamente determinada de antemano por ellos y ellas. Asimismo, debemos mencionar que muy pocos estudiantes relatan una toma de decisiones conjunta con sus padres/madres:

Mi madre y mi padre siempre me dijeron que lo que quisiera. Lo que yo haga, ellos lo aceptan (Mujer, GD1).

Consultar con mis padres sí, pero la última palabra la tengo yo, porque yo soy el que voy a tener que estudiar (Varón, GD2).

No me dieron opinión ninguna porque no la pedí, voy a hacer lo que yo quiera (Mujer, GD2).

Me dejaron a mi elección el bachiller y me apoyan en que estudie lo que me guste a mí (Varón, GD3).

No me dicen que escoja una cosa ni otra. Lo dejan a mi elección (Varón, GD4).

Tanto mi padre como mi madre me han dejado escoger lo que quiera y me han apoyado (Mujer, GD5).

Los progenitores, en general, refrendan las afirmaciones de los estudiantes al considerar que la elección realizada es una responsabilidad de los hijos e hijas y prefieren no interferir en ello. Algunos de ellos aclaran que no imponen nada como obligatorio ni les presionan en ningún sentido y se indica que su misión es actuar de apoyo a las decisiones que toman:

Yo concretamente no he influido nada en lo que quieren hacer (Mujer, GD2). 
$\mathrm{M}^{\mathrm{a}}$ del Carmen Rodríguez Menéndez et al. Esto es lo que me gusta y lo que voy a estudiar”...

No sé, yo nunca le inculqué nada, ni le dije haz esto o haz lo otro, jamás (Mujer, GD1).

El mayor dijo que iba al tecnológico pues siempre fue de ideas muy claras, y muy suyas...no creo que hayamos intervenido ni el padre ni yo en la decisión tomada (Mujer, GD1).

Sin lugar a dudas, de lo visto hasta el momento debemos hacer una reflexión: ¿Los progenitores tienen tan poca influencia en la elección académica como parece desprenderse de las palabras de los adolescentes o esta percepción no se corresponde con una realidad en la que los padres y madres influyen más de lo que los implicados están dispuestos a admitir?. Podemos poner un ejemplo paradigmático de tal situación. Es el caso de S., alumno brillante con notas excelentes que no sabe si realizar un módulo de mecánica o una ingeniería. Sus docentes y progenitores le apoyan y refuerzan para que estudie una carrera universitaria pero él manifiesta una preferencia por lo manual unida a un escaso autoconcepto referido a sus posibilidades para finalizar con éxito una ingeniería. Su padre es camionero y su madre trabaja en un supermercado. Su padre ha actuado como modelo de referencia para él, le gusta el trabajo de su padre pero reconoce que es muy esclavo: "A mi me gusta mucho el trabajo de mi padre, pero veo que es una basura, entonces no voy a por el trabajo de mi padre porque veo que es esclavizado, pero a mí me gusta mucho". En otro contexto y situación distinta, quizás hubiese sido un chico que se hubiese decantado por esta profesión; no obstante, el refuerzo parental- y docente- para que realice estudios superiores, su consejo y apoyo le hacen decantarse por la universidad. A pesar de ello cuando se le pregunta por qué ha elegido el bachillerato tecnológico, manifiesta que: "Hago lo que quiero. Mis padres me apoyan en lo que haga, pero el que sabe lo que hay soy yo y yo elijo”. El apoyo y refuerzo parental ha sido importante pero él considera que las decisiones las adopta individualmente.

Finalmente debemos mencionar que cuando los estudiantes reconocen una clara influencia de un progenitor más que otro, la mayoría indican la mayor influencia materna. Además, podemos observar que lo importante para ellos y ellas es que los progenitores les apoyen en aquello que desean hacer. La forma en que transcurren las conversaciones con el alumnado deja entrever la idea de que la decisión sobre los estudios es personal y que los padres/madres están para apoyar lo que ellos y ellas ya han elegido:

La persona que más me apoya es mi madre (Mujer, GD1).

Mi madre siempre me ha apoyado en mis elecciones y aunque yo he querido hacer el bachiller me obligaba a hacerlo porque decía que era la única salida que, a día de hoy, vale (Mujer, GD5).

Mi madre siempre me apoyó en cualquier decisión (Varón, GD5). 
$\mathrm{M}^{\mathrm{a}}$ del Carmen Rodríguez Menéndez et al. Esto es lo que me gusta y lo que voy a estudiar”...

\section{El profesorado y su apoyo a la elección académica}

En ocasiones, el profesorado ha sido una fuente de apoyo en el proceso de elección académica. No obstante, nos llama la atención que en todos los grupos de discusión se preguntó explícitamente por el apoyo recibido por los docentes y pocos estudiantes fueron capaces de relatar una experiencia de apoyo importante durante la educación secundaria. Es más, hubo grupos de discusión en los que, de forma prácticamente unánime, se declaró que no existía apoyo docente para la toma de decisiones académicas. Así como la influencia parental se reconoce, aunque siempre pasada por el tamiz de la decisión personal y la libertad individual, hay una menor percepción de las influencias y apoyos que reciben de sus docentes, si bien hay excepciones a la regla:

El tutor de este año siempre nos apoya en todo, o sea, la carrera (Mujer, GD1).

Yo quería hacer medicina, pero vivía en mi mundo creyendo que lo podía hacer hasta que P. (profesor de química) me dijo que no, que no lo iba a poder hacer... aunque me dijera que no, lo hizo por mi bien (Varón, GD2).

Ahora en $2^{\circ}$ de bachiller donde más te tendrían que apoyar para decir..., porque hay tiempo de sobra, hay tiempo para dar materia pero también se pierde mucho tiempo en otras cosas, debería haber tiempo para hablar de lo que podemos hacer en un futuro y muchas horas de tutoría también que es donde se debería de hacer están perdidas (Varón, GD3).

De forma complementaria, lo que sí indicaron los estudiantes fue el discurso motivador que manejan algunos docentes y que genera interés por su materia. En este sentido, sí reconocen la influencia docente en la determinación de la preferencia por algunas materias, que, a su vez, puede orientar su carrera académica. Esta influencia que, a priori, es positiva también provoca la sensación de que, en ocasiones, sus elecciones académicas son azarosas y dependen del interés que algunos docentes han creado por determinadas materias (véase el primer fragmento discursivo de los que se presentan a continuación). Estas opiniones son refrendadas por muchos progenitores, quienes indican que un docente puede inclinar a sus alumnos hacia determinadas materias:

En $3^{\circ}$ de la ESO tuve una profesora de fisica y química que solo estuvo aquí un año que hizo que odiara esa asignatura, entonces en $4^{\circ}$ de la ESO en vez de escoger física y química escogí latín y todo de letras por eso pensaba que iba a ir por ese bachiller, y al final tuve ese año un profesor bueno de matemáticas y escogí el tecnológico en el último momento (Mujer, GD4).

Para mí influyó mucho una profesora de tecnología, y por eso escogi ese bachillerato (Varón, GD5).

Pero también es cierto que este año influyó mucho el profesor que tiene mi hija de tecnología, el año pasado cuando empezó con todo lo que es lo eólico...eso le encantó, es más, estamos poniendo en casa placas solares, ella fue para allá a mirar, esto funciona así de esta forma. Entonces muchas veces, no sólo es cuestión 
$\mathrm{M}^{\mathrm{a}}$ del Carmen Rodríguez Menéndez et al. Esto es lo que me gusta y lo que voy a estudiar”...

de lo que quieran, es también que encuentres un profesor que te haga ver algo que te guste (Mujer, GD1).

Los amigos y amigas en el proceso de elección académica

Respecto a los amigos y amigas, la mayoría manifiesta que no han influido en su decisión de estudiar el bachillerato científico-tecnológico. Nuevamente vuelve a salir la idea de que las decisiones vocacionales son individuales. Lo que sí afirman es que los amigos y amigas han sido un apoyo en el proceso, pero cuando la decisión ya estaba tomada, no antes. Además, se manifiesta una creencia rotunda en el apoyo de los amigos y amigas:

Tampoco me influyeron mis amigas, aunque al final una de ellas está conmigo en clase (Mujer, GD4).

Yo particularmente lo elegí porque fue lo que me gustaba no por los amigos. A mí no me influyó para nada (Varón, GD4).

No me influyeron para nada (2 varones, GD1).

Salgo con amigas del bachiller de sociales y humanidades y a mí sí me motivan, me dicen: "Venga que vas aprobar, que al final la selectividad bien, tú puedes porque siempre estudias mucho” Siempre me están apoyando (Mujer, GD3).

Los amigos siempre te van a apoyar (Mujer, GD3).

Nuevamente nos volvemos a hacer la pregunta acerca de la verdadera influencia que tienen sus compañeros y compañeras. En este sentido, resulta paradigmático el siguiente extracto discursivo ya que no deja de ser inquietante la posibilidad de que procesos de decisión académica tan importantes como es la elección de la modalidad de bachillerato se realicen sobre la base de argumentaciones tan débiles. Si esto es así, no nos queda sino concluir que algo está fallando en el sistema educativo:

Alumno.- Mis amigos no influyeron para nada, pero sabía que iban a escoger lo mismo que yo porque ya habíamos comentado la jugada antes de comenzar el bachiller.

Entrevistadora.- O sea, que la mayoría estáis juntos.

Alumno: Dije: "Voy a coger este bachiller así que vosotros hacer lo que os dé la gana"

Entrevistadora: Y te siguieron.

Alumno: No sé si me siguieron o no.

Entrevistadora: Pero están haciendo lo mismo que tú.

Alumno: Estamos todos de casualidad. 
$\mathrm{M}^{\mathrm{a}}$ del Carmen Rodríguez Menéndez et al. Esto es lo que me gusta y lo que voy a estudiar”...

Respecto a las opiniones de los padres, hemos encontrado ideas muy variadas respecto al papel del grupo de iguales, desde aquellos que opinan que no tienen ninguna influencia hasta la percepción de que su influjo ha sido determinante:

Yo creo que los amigos influyen en muchas cosas, pero para el mío para elegir carrera no (Mujer, GD1).

Escogió ese porque estaban sus amigos, o sea digo pero R., pero si tú, "no porque está Juanito, Fulanito, Menganito” y digo pues hala, tú mismo (Mujer, GD2).

\section{Conclusiones}

Nuestros resultados son coincidentes con los obtenidos por Santana y Feliciano (2009. Véase también Cortés y Conchado, 2012) quienes constatan que el bachillerato sigue siendo considerado la puerta de acceso a la universidad y no a la formación profesional de grado superior. Asimismo, podemos concluir que progenitores y estudiantes coinciden al afirmar que la principal razón que dirige la elección es la preferencia o el gusto por determinadas materias (véase también Cortés y Conchado, 2012; García, Padilla y Suárez, 2009). Esta razón prevalece sobre otras como las salidas profesionales o el rendimiento académico previo.

Asimismo, la mayoría de los estudiantes se muestran confiados y seguros sobre la elección y aparentan una seguridad que incluso se proyecta sobre sus posibilidades de éxito académico en la Universidad, opción que encuentran más asequible que el bachillerato porque opinan que estudiar lo que gusta (carrera universitaria) hará más atractivo y, por tanto, más fácil el aprendizaje. Sin embargo, los padres y madres no corroboran esta confianza y seguridad pues argumentan que incluso en bachillerato muchos estudiantes manifiestan indecisión ante su futuro académico y que toman decisiones poco fundamentadas. Desde esta perspectiva, nuestros resultados son coincidentes con los obtenidos por Santana y Feliciano (2009), quienes concluyen que si bien el alumnado de su muestra se siente relativamente seguro con la opción académica elegida en el bachillerato, la toma de decisiones adolece de falta de rigor y de una adecuada evaluación de las posibilidades reales de cada estudiante (véase también García, Padilla y Suárez, 2009).

Los estudiantes afirman, sin ningún género de dudas, que las decisiones académicas que adoptan son personales y han sido tomadas, en la mayoría de los casos, de manera individual. En este sentido, reconocen que el estímulo y apoyo de sus progenitores es fundamental para asegurarse de que toman la decisión correcta, pero no dejan de resaltar que la decisión última está en sus manos. Las opiniones manifestadas por los estudiantes en este sentido, también son corroboradas por los padres y madres. Por tanto, podemos concluir que el apoyo parental es un factor clave en el desarrollo de la carrera de estos estudiantes, pero es un apoyo que se manifiesta fundamentalmente en la necesidad de saber que sus padres/madres se interesan por ellos y confían en que sabrán tomar buenas decisiones (véase también Cortés y Conchado, 2012; Fisher y Padmawidjaja, 1999; Paa y McWhirter, 2000; Schultheiss et al., 2001). Asimismo, y de forma similar a otras investigaciones (Nauta y Kokaly, 2001; Otto, 2000; Wycoff, 
1996) en aquellos casos en que se nombra a un progenitor como una figura relevante en el proceso de decisión académica, en la mayoría de las ocasiones se nombra a las madres.

Asimismo, y a diferencia de otros estudios (Fouad et al., 2010; Flores y Obasi, 2005; Nauta y Kokaly, 2001; Carvalho y Taveira, 2014) hay una percepción mucho menor de que los docentes influyen en la toma de decisiones académicas. Los estudiantes perciben que su influencia es menor que la que tienen los progenitores (véase Paa y McWhirter, 2000) y no indican que reciban asesoramiento vocacional por parte de ellos, ni siquiera en la hora de tutorías que podría estar destinada, entre otras cosas, para tal fin (véase Santana y Feliciano, 2008). A pesar de ello, estudiantes y progenitores reconocen que determinados docentes pueden influir en la decisión de una forma indirecta porque con su metodología didáctica logran interesar a sus estudiantes en la materia.

Finalmente, nuestros resultados no son concluyentes respecto al papel que desempeña el grupo de iguales en las decisiones académicas. A diferencia de otros estudios (Ali, McWhirter y Chronister, 2005; Dietrich y Kracke, 2009; Kenny et al., 2003; Ma y Yeh, 2010; McWhirter et al., 2007; Otto, 2000; Schultheiss et al., 2001) los estudiantes afirman sentirse apoyados por sus amigos y amigas en sus decisiones pero indican que no han influido en una decisión que, se vuelve a insistir, es adoptada de forma individual. No obstante, los discursos de los progenitores manifiestan más dudas respecto a la posible influencia del grupo de iguales en la toma de decisiones vocacional.

Queremos concluir afirmando que los resultados de esta investigación, junto con los obtenidos en estudios realizados anteriormente, tienen importantes implicaciones para la práctica de la orientación educativa. Los estudiantes fundamentan sus decisiones académicas sobre la base de la preferencia y gusto personal por determinada opciones, información que es corroborada por sus progenitores. Por ello creemos necesario, de acuerdo con Santana, Feliciano y Jiménez (2009. Véase también Paa y McWhirter, 2000; Santana, Feliciano y Santana, 2013), que los estudiantes incrementen su autoconocimento, siendo conscientes de sus potencialidades y limitaciones y adoptando decisiones consecuentes que no solo se fundamenten en una pretendida vocación. En este sentido, y siendo conscientes de que en última instancia es el estudiante el que ha de tomar sus propias decisiones, creemos que las instituciones educativas deben preparar y orientar al alumnado en un proceso de gran relevancia para su vida. Así es preciso que en los primeros años de la educación secundaria se den a los estudiantes las herramientas para favorecer su autoconocimento y se les provea de información acerca de las opciones académicas y laborales que les permitan adoptar una decisión fundamentada y racional.

Al mismo tiempo, queremos resaltar la influencia que pueden y deben jugar los progenitores y los docentes en el proceso de decisión académica. De este modo, y como consideran Santana, Feliciano y Jiménez (2009) estos agentes socializadores deben ayudar al alumnado a tomar decisiones sustentadas en el principio de realidad y ajustadas a sus intereses y capacidades. Por tanto, los programas de orientación en los contextos escolares deberían implicar a miembros de la familia, particularmente a 
$\mathrm{M}^{\mathrm{a}}$ del Carmen Rodríguez Menéndez et al. Esto es lo que me gusta y lo que voy a estudiar”...

padres/madres, y a sus docentes porque esos adultos están presentes en los planes académicos y en las decisiones vocacionales de los estudiantes (Flores y Obasi, 2005).

Del mismo modo, Paa y Mcwhirter (2000. Véase también Fisher y Padmawidjaja, 1999; Turner, Stewart y Lapan, 2004) indican que los orientadores de los centros educativos de secundaria deben articular acciones para informar a los padres/madres de las decisiones académicas que deberán tomar sus hijos e hijas en la educación secundaria y deben dotarles de las herramientas necesarias para abordar con ellos conversaciones acerca de su futuro académico y laboral. En línea similar, estos autores también recomiendan que los orientadores trabajen más estrechamente con los docentes para hacerles más conscientes de su papel en las decisiones académicas de sus estudiantes y para dotarles de las estrategias necesarias para ejercer una adecuada labor de tutoría en este ámbito.

\section{Referencias bibliográficas}

ALI, S.; MCWHIRTER, E. Y CHRONISTER, K. (2005). Self-efficacy and vocational outcome expectations for adolescents of lower socioeconomic status. A pilot study. Journal of Career Assessment, 13(1), 40-58.

CARVALHO, M. Y TAVEIRA, M. (2014). El papel del profesorado en la toma de decisiones académico y profesionales del alumnado. Revista Española de Orientación y Psicopedagogía, 25(3), 20-35.

CORTÉS, A. Y CONCHADO, A. (2012). Los contextos parentales y académicos y los valores laborales en la toma de decisiones en bachillerato. Estudios sobre Educación, 22, 93-114.

DIETRICH, J. Y KRACKE, B. (2009). Career-specific parental behaviors in adolescents' development. Journal of Vocational Behavior, 75, 109-119.

FISHER, T. Y PADMAWIDJAJA, I. (1999). Parental influences on career development perceived by African American and Mexican American college students. Journal of Multicultural Counselling y Development, 27(3), 1-18.

FOUAD, N.A. ET AL. (2010). Barriers and supports for continuing in mathematics and science: gender and educational level differences. Journal of Vocational Behavior, 77, 361-373.

GARCÍA, S.; PADILLA, T. Y SUÁREZ, M. (2007). Los intereses académicos y profesionales de chicas que finalizan la escolaridad obligatoria. Revista de Educación, 349, 311-334.

KELLER, B. Y WHISTON, S. (2008). The role of parental influences on young adolescent's career development. Journal of Career Assessment, 16(2), 198-217.

KENNY, M. ET AL. (2003). The role of perceived barriers and relational support in the educational and vocational lives of urban high school students. Journal of Counselling Psychology, 50(2), 142-155. 
$\mathrm{M}^{\mathrm{a}}$ del Carmen Rodríguez Menéndez et al. Esto es lo que me gusta y lo que voy a estudiar”...

MA, P. Y YEH, C. (2010). Individual and familial factors influencing the educational and career plans of Chinese immigrants youths. The Career Development Quarterly, 58, 230-245.

MAU, W.; HITCHCOCK, R. Y CALVERT, C. (1998). High school students' career plans: the influence of others' expectations. Professional School Counseling, 2(2), 161-166.

MCWHIRTER, E. H. ET AL. (2007). Perceived barriers and postsecondary plans in Mexican American and White adolescents. Journal of Career Assessment, 15(1), 119-138.

NAUTA, M. Y KOKALY, M. (2001). Assessing role model influences on students' academic and vocational decisions. Journal of Career Assessment, 9(1), 81-99.

OTTO, L. B. (2000). YOUTH PERSPECTIVES ON PARENTAL CAREER INFLUENCE. JOURNAL OF CAREER DEVELOPMENT, 27(2), 111-118.

PAA, H. K. Y MCWHIRTER, E. (2000). Perceived influences on high school students' current career expectations. The Career Development Quarterly, 49, 2944.

PERRONE, K. ET AL. (2002). Role model influence on the career decidedness of college students. College Student Journal, 36(1), 109-112.

SANTANA, L. Y FELICIANO, L. (2009). Dificultades en el proceso de toma de decisiones académico-profesionales: el reto de repensar la orientación en Bachillerato. Revista de Educación, 350, 323-350.

SANTANA, L. Y FELICIANO, L. (2011). Percepción de apoyo de padres y profesores, autoconcepto y toma de decisiones en bachillerato. Revista de Educación, 355, 493-519.

SANTANA, L.; FELICIANO, L. Y JIMÉNEZ, A. (2009). Autoconcepto académico y toma de decisiones en el alumnado de bachillerato. Revista Española de Orientación y Psicopedagogía, 20(1), 61-75.

SANTANA, L.; FELICIANO, L. Y SANTANA, J. (2013). Madurez y autoeficacia vocacional en $3^{\circ}$ y $4^{\circ}$ de ESO, bachillerato y ciclos formativos. Revista Española de Orientación y Psicopedagogía, 24(3), 8-26.

SCHULTHEISS, D. ET AL. (2001). Relational influences in career development: a qualitative inquiry. The Counseling Psychologist, 29(2), 216-239.

TURNER, S.; STEWARD, J. Y LAPAN, R. (2004). Family factors associated with sixth-grade adolescents' math and science career interests. The Career Development Quarterly, 53, 41-51.

VALLES, M. S. (2000). Técnicas cualitativas de investigación social. Reflexión metodológica y práctica profesional. Madrid: Síntesis. 
$\mathrm{M}^{\mathrm{a}}$ del Carmen Rodríguez Menéndez et al. Esto es lo que me gusta y lo que voy a estudiar"...

WYCOFF, S. (1996). Academic performance of Mexican American women: sources of support that serve as motivating variables. Journal of Multicultural Counselling $y$ Development, 24(3), 146-155.

\section{Correspondencia con los autores}

$\mathrm{M}^{\mathrm{a}}$ del Carmen RODRÍGUEZ MENÉNDEZ

Facultad de Formación del Profesorado y Educación. Despacho 322

C/ Aniceto Sela, s.n.

33005 Oviedo (España)

e-mail: carmenrm@uniovi.es

José Vicente PEÑA CALVO

Facultad de Formación del Profesorado y Educación. Despacho 322

C/ Aniceto Sela, s.n.

33005 Oviedo (España)

e-mail: vipe@uniovi.es

$\mathrm{M}^{\mathrm{a}}$ de las Mercedes INDA CARO

Facultad de Formación del Profesorado y Educación. Despacho 314

C/ Aniceto Sela, s.n.

33005 Oviedo (España).

e-mail: indamaria@uniovi.es 